\title{
Using oncology real-world evidence for quality improvement and discovery: the case for ASCO's CancerLinQ
}

\author{
Robert S Miller ${ }^{1}$ \& Jennifer L Wong*,1 \\ ${ }^{1}$ American Society of Clinical Oncology, CancerLinQ, Alexandria, VA, USA \\ * Author for correspondence: Jennifer.Wong@cancerlinq.org
}

\begin{abstract}
'CancerLinQ offers a set of electronic quality performance indicators based on existing Quality Oncology Practice Initiative measures, which return concordance rates by firing against EHR data. Additional apps include a data exploration/cohort expansion tool, data visualization tools and a dynamically generated timeline display of key diagnostic and treatment milestones for each patient's course”
\end{abstract}

First draft submitted: 6 October 2017; Accepted for publication: 9 October 2017; Published online: 20 October 2017

Keywords: ASCO $\bullet$ big data $\bullet$ CancerLinQ $\bullet$ quality $\bullet$ real-world evidence $\bullet$ research

In recent years, the field of oncology has been characterized by remarkable diagnostic and therapeutic promise accompanied by astonishing complexity. Recognizing this precipitous rate of change, in 2012 the board of directors of the American Society of Clinical Oncology (ASCO) released a vision statement of what cancer care might look like 25 years in the future in order to better prepare the field. In 'Shaping the Future of Oncology: Envisioning Cancer Care in 2030' [1], the board proposed that three key drivers would have the biggest impact:

- 'Big data'

- Cancer panomics

- Delivering value

Advances in health information technology would enable clinical, administrative and patient-generated data on virtually every patient with cancer to be systematically collected, analyzed and shared, in near real time, to immediately impact patient care and contribute to the generation of new knowledge. Cancer treatment would come to be based on personalized, data-informed insights delivered through clinical decision support instead of static clinical practice guidelines. In addition, revolutions in big data and molecular medicine would reduce today's fragmentation and lead to an oncology care delivery system that is truly patient-centric and global.

To operationalize this vision, and informed by prior work of the Institute of Medicine [2], ASCO set forth to create a learning health system for oncology by developing the big data, health technology system Cancer Learning Intelligence Network for Quality (CancerLinQ ${ }^{\circledR}$, CancerLinQ LLC, VA, USA) [3]. Built and implemented by CancerLinQ LLC, a wholly owned, nonprofit subsidiary of ASCO, CancerLinQ is now a reality, having gone live in oncology practices throughout the USA in 2016. CancerLinQ aggregates data from electronic health records (EHRs) via direct feeds, with no requirement for the clinician to capture the data in a specific format, and then processes it through a series of cloud-based databases, where it undergoes progressive transformation and deidentification. The data are made available to subscribing practices - in other words, those centers contributing data to the platform - through applications accessed via a web portal. CancerLinQ is an extension of ASCO's portfolio of quality programs, most notably its signature Quality Oncology Practice Initiative [4]. CancerLinQ offers a set of electronic quality performance indicators based on existing Quality Oncology Practice Initiative measures, which return concordance rates by firing against EHR data. Additional apps include a data exploration/cohort expansion tool, data visualization tools and a dynamically generated timeline display of key diagnostic and treatment milestones for each patient's course [5].

Future 8 Medicine 
Managing the heterogeneous nature of EHR data to achieve the highest possible data quality is the single biggest obstacle facing platforms like CancerLinQ. Unlike clinical trial data which conform to the strict conditions enabled by prespecified patient eligibility criteria, treatment regimens and diagnostic assessments, transactional EHR data are inherently less organized and frequently not recorded in discrete fields, especially for many critical oncologic variables such as cancer stage, biomarkers, adverse event documentation and disease progression. The lack of a common data model for the oncology record is another challenge. CancerLinQ has compensated for this, in part, by transforming raw EHR data using ontology services based on the National Cancer Institute (NCI) Metathesaurus [6] and other vocabularies, along with a series of custom software rules, now numbering in the tens of thousands, to organize it into an internal data model, based on the quality data model from the Centers for Medicare and Medicaid Services [7]. Nonetheless, data elements found only in unstructured documents such as clinician notes must be retrieved through human curation (manual data abstraction), enhanced by natural language processing. As a result, important gaps in data will continue to exist.

The observational data in the CancerLinQ platform represents 'real-world data' (RWD), defined as a source other than a clinical trial, such as EHR data, registries, insurance claims, prescription refills, death indices and patientgenerated health data. Real-world evidence (RWE), derived from RWD, can be enormously valuable to complement traditional data sources, despite concerns about quality and provenance. Although the role of randomized clinical trials in generating evidence is unquestioned, given their strong internal validity and minimization of bias, their disadvantages are widely recognized. Conversely, RWE may have greater external validity since it reflects 'what does work' (effectiveness), not merely 'what can work' (efficacy), despite a greater susceptibility to selection bias, performance bias and other challenges [8]. Not only is there growing interest in the applicability of RWE to industry and regulatory end points [9], RWE is highly relevant to the quality improvement and clinical care functions of tools like CancerLinQ. In addition, the feedback loop of the oncology learning health system that CancerLinQ is enabling will use RWE outcomes to inform the development of clinical practice guidelines in a rapid and iterative fashion, to increase guideline relevance and narrow evidence gaps.

The aspiration of CancerLinQ to build a real world, big data learning system extends far beyond its growing network of $100+$ community oncology practices, academic institutions and major health systems representing thousands of oncologists across the USA [10]. In order to offer a more representative, holistic view of the cancer patient's journey to support quality improvement efforts and discovery, CancerLinQ is tapping into information that exists beyond the limited cohort of data within traditional clinical trials and the lens of medical oncology alone [11]. Thus, CancerLinQ has engaged the broader oncology community beyond ASCO's membership to incorporate the integral perspectives of the entire oncology care team, all in an effort to create one of the largest, most robust sources of RWE in oncology.

Committed to building coalitions of support and key strategic alliances with patients, providers and partners, CancerLinQ has taken determined strides to expand its scope, reach and impact [12]. CancerLinQ has formally partnered with public, private and not-for-profit institutions representing a wide spectrum of forward-thinking leaders and organizations. These foundational partners offer thought leadership and support from their membership base of influencers and experts, all of whom are stakeholders impacting key points in a given patient's cancer care journey.

As a well-positioned enabler and accelerator of a variety of data initiatives, in 2016 CancerLinQ drew attention and support from the White House National Cancer Moonshot Initiative, led by then vice president Joseph Biden. Championing collaboration and data sharing to catalyze faster progress toward cures, CancerLinQ was heralded by the Vice President as an opportunity to address the need for 'open data, open collaboration' to facilitate the creation and sharing of new discoveries to improve care [13]. One of the promising efforts underway is a NCI-CancerLinQ data sharing alliance to support the NCI's Surveillance, Epidemiology and End Results program, one of the primary sources of data on cancer incidence and survival in the USA.

The CancerLinQ Oncology Leadership Council (OLC) was launched as the official body of strategic advisors comprising member representatives from CancerLinQ's official founding partner organizations and advisory groups, including professional medical specialty societies, government agencies and industry [14]. Currently, the CancerLinQ OLC is a national consortium including the US FDA, NCI, American Academy of Physician Assistants, American Society for Radiation Oncology, Cancer Informatics for Cancer Centers, College of American Pathologists, Hematology/Oncology Pharmacy Association, Oncology Nursing Society, Society of Gynecologic Oncology and AstraZeneca. These partners help CancerLinQ address current challenges in healthcare delivery and work collectively to improve treatment and patient outcomes in a timely fashion. 
To drive RWE-enabled research and discovery in oncology, in 2016 ASCO announced the development of CancerLinQ Discovery ${ }^{\text {TM }}$ (CancerLinQ ${ }^{\circledR}$, CancerLinQ LLC, VA, USA) - highly curated, de-identified, fit-forpurpose sets of clinical data - as a public resource available to the research community, including but not limited to members of the CancerLinQ OLC, academic and community researchers, public health agencies, life sciences organizations and patient advocacy groups [15]. Interested parties may submit requests to the CancerLinQ Discovery Research \& Publications Committee - a volunteer, scientific advisory committee charged with reviewing and approving data requests [16] - for access to customized and statistically de-identified real-world datasets. Further details about the request process and a copy of the data dictionary are available on the CancerLinQ website [17].

The goal of leveraging CancerLinQ Discovery is to create new knowledge from RWD generated from everyday practice to improve patient outcomes. Identifying treatment options for patients with rare tumor types lacking clinical trial data or informing decision making about a commonly prescribed treatment in a patient population with comorbidities that would have otherwise excluded them from clinical trials of the same agent are two use cases. The FDA-CancerLinQ collaboration will leverage CancerLinQ's ability to harness data from patient populations not well represented in traditional research protocols and "explore a variety of issues, including the optimal sequence of treatments, the impact that other health problems have on treatment tolerability and cancer outcomes, and the experience with immunotherapy combinations versus single agents" ${ }^{118]}$. Investigators will initially focus on the role of checkpoint inhibitors in melanoma and other cancers.

Managing the vast amount of data required to provide high-quality cancer care in 2017 and ensuring that the knowledge base available to all members of the oncology ecosystem grows in commensurate fashion require information systems and new paradigms that were unforeseen just a few years ago. The CancerLinQ learning health system is one potential solution to meet this challenge and in doing so extends ASCO's primary mission of delivering education, promoting research and enabling quality care for patients everywhere.

\section{Financial \& competing interests disclosure}

The authors are employees of ASCO/CancerLinQ. The authors have no other relevant affiliations or financial involvement with any organization or entity with a financial interest in or financial conflict with the subject matter or materials discussed in the manuscript apart from those disclosed.

No writing assistance was utilized in the production of this manuscript.

\section{Open access}

This work is licensed under the Attribution-NonCommercial-NoDerivatives 4.0 Unported License. To view a copy of this license, visit http://creativecommons.org/licenses/by-nc-nd/4.0/

\section{References}

1 American Society of Clinical Oncology. Shaping the future of oncology: envisioning cancer care in 2030. www.asco.org/sites/new-www.asco.org/files/content-files/about-asco/documents/2030-shaping-future-oncology-cancer-care.pdf.

2 Abernethy AP, Etheredge LM, Ganz PA et al. Rapid learning system for cancer care. J. Clin. Oncol. 28, 4268-4274 (2010).

3 Schilsky RL, Miller RS. Creating a learning health care system in oncology. In: Oncology Informatics. Hesse BW, Ahern DK, Beckjord E (Eds). Elsevier, Amsterdam, The Netherlands (2016).

4 Blayney DW, McNiff K, Eisenberg PD et al. Development and future of the American Society of Clinical Oncology's Quality Oncology Practice Initiative. J. Clin. Oncol. 32, 3907-3913 (2014).

5 Miller RS. CancerLinQ update. J. Oncol. Pract. 12, 835-837 (2016).

6 National Cancer Institute. NCI Metathesaurus. https://ncimeta.nci.nih.gov/ncimbrowser.

7 eCQI Resource center. What is the quality data model. https://ecqi.healthit.gov/qdm

8 Schilsky RL. Finding the evidence in real-world evidence: moving from data to information to knowledge. J. Am. Coll. Surg. 224, 1-7 (2017).

9 Sherman RE, Anderson SA, Dal Pan GJ et al. Real-world evidence - what is it and what can it tell us? N. Engl. J. Med. 375, 2293-2297 (2016).

10 West Cancer Center joins CancerLinQ ${ }^{\circledR}$ as participating practice. www.asco.org/about-asco/press-center/news-releases/west-cancer-center-joins-cancerlinq-participating-practice

11 Donato C. How will personalized medicine shift from great concept to reality? www.forbes.com/sites/sap/2016/10/25/how-will-personalized-medicine-shift-from-great-concept-to-reality/\#5743bff01 e60 
12 Cavallo J. How ASCO’s CancerLinQ Discovery ${ }^{\text {TM }}$ will help speed research advances and improve patient outcomes. www.ascopost.com/ issues/april-25-2017/how-asco-s-cancerlinq-discovery-will-help-speed-research-advances-and-improve-patient-outcomes.

13 ASCOcancer. Vice President Joe Biden's Moonshot address at the 2016 ASCO Annual Meeting. www.youtube.com/watch? $=$ WGXrCerRQUw\&list=WL\&index $=44$.

14 Fitzpatrick K. The newest members of CancerLinQ's community-FDA and NCI. www.ajmc.com/journals/evidence-based-oncology/ 2017/August-2017/The-Newest-Members-of-CancerLinQs-Community-FDA-and-NCI.

15 Newly Developed CancerLinQ Discovery ${ }^{\text {TM }}$ translates big data into practical knowledge for cancer community (2016). www.asco.org/about-asco/press-center/news-releases/newly-developed-cancerlinq-discovery-translates-big-data.

16 CancerLinQ Discovery ${ }^{\text {TM }}$ to begin accepting research proposals on October 1. www.asco.org/advocacy-policy/asco-in-action/cancerlinq-discovery-begin-accepting-research-proposals-october-1.

17 ASCO CancerLinQ Discovery ${ }^{\mathrm{TM}}$. http://cancerlinq.org/research-database

18 [No authors listed]. FDA partners with ASCO’s CancerLinQ. Cancer Discov. 7(8), OF2 (2017). 